\title{
Binary colloidal glasses under transient stress- and strain-controlled shear
}

\author{
Tatjana Sentjabrskaja and Jan Hendricks ${ }^{\text {a) }}$ \\ Condensed Matter Physics Laboratory, Heinrich Heine University, 40225 Düsseldorf, Germany
}

Alan R. Jacob ${ }^{\mathrm{b})}$ and George Petekidis

IESL-FORTH and Department of Materials Science and Technology, University of Crete, Heraklion, Greece

Stefan U. Egelhaaf ${ }^{\text {() }}$

Condensed Matter Physics Laboratory, Heinrich Heine University, 40225 Düsseldorf, Germany

Marco Laurati

Division de Ciencias e Ingenieria, Universidad de Guanajuato, 37150 Leon, Mexico

(Received 11 January 2017; final revision received 7 July 2017; published 16 November 2017)

\begin{abstract}
We investigate the yielding and transition to flow of different colloidal glasses. Using a single model system, a binary mixture of colloidal hard spheres with different compositions and size ratios, we study single, double and asymmetric glasses, which differ in the degree of mobility of the small particles and the caging mechanisms of the large spheres. The rheological response following either a step to a constant shear rate or to a constant stress (creep) is measured and the two responses are quantitatively compared. Although the same steady state of flow is observed at long times, the transient responses in strain- and stress-controlled experiments differ significantly. To achieve yielding and a steady state of flow, less time and less energy input is required if a constant strain rate is applied. Moreover, larger strain rates or stresses result in faster yielding and flow, but require more total energy input. If a constant strain rate is applied, yielding and the transition to flow depend on the properties of the glass state, while much smaller differences are observed if a constant stress is applied. C 2017 The Society of Rheology. https://doi.org/10.1122/1.5009193
\end{abstract}

\section{INTRODUCTION}

The behavior of glasses under application of a mechanical deformation or a force, that is under shear, is of great relevance for many applications that rely on or exploit the flow of glass-forming systems. Colloidal glasses have proven to be ideal model systems to study this behavior $[1,2]$. They are characterized by arrested dynamics resulting in a very slow internal relaxation. Dynamical arrest occurs at large volume fractions and is associated with crowding [1-4]; particles are trapped in "cages" formed by their nearest neighbors, at least until activated processes can restore diffusion [5]. External driving, e.g., by application of shear, leads to a competition between the slow internal relaxation and the time scale imposed by shear. At small deformations, where the linear viscoelasticity of the system is probed, the response of colloidal glasses is dominated by elasticity and the system behaves as a viscoelastic solid [6-10]. In contrast, under

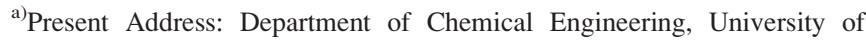
Leuven, KU Leuven, 3001 Leuven, Belgium.

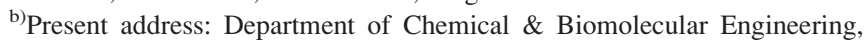
North Carolina State University.

c) Author to whom correspondence should be addressed; electronic mail: stefan.egelhaaf@uni-duesseldorf.de

large shear deformations the system shear melts and starts to flow with the viscous instead of the elastic component dominating its response [10-13].

Continuous shear, namely, the application of a constant strain rate or a constant stress, leads to steady flow beyond the yield point [9-11,13-20]. The transition from rest and solidlike behavior to steady flow is characterized by transient phenomena [12,19-21]. At rest, the behavior of the investigated glasses is dominated by cages formed by neighbors that, on average, are isotropically distributed. After application of a step in strain rate, the cages become deformed and the corresponding convection of the cages is associated with superdiffusive dynamics at intermediate times. Thus, an anisotropy in the local microscopic structure develops. The cage deformation can be quantified based on the pair distribution function $g(r)$. The deformations are particularly evident in the sheargradient plane $[12,19,20]$. The maximum cage deformation coincides with the occurrence of a stress overshoot in the rheological response [12,19-22]. In the steady state, the shearinduced longest relaxation time is found to be inversely proportional to the shear rate reflecting a convective cage release mechanism through which cages break and particles rearrange [23]. A constant stress also leads to the fluidization of the glass if the stress is larger than the yield stress [10,13-15,24,25]. In contrast, for stresses below the yield stress, the system does not 
flow [14,15, 26-29]. Instead, a creep regime is observed which is characterized by a slow sublinear increase of the strain as a function of time. The strain is linearly related to the mean squared displacement (MSD) of the individual particles [24]. This finding can be related to the observation that, under stress-control, the single-particle dynamics as well as the strain are dominated by groups of highly mobile particles. The fusion of regions with highly mobile particles eventually leads to flow [24]. In contrast, in the straincontrolled case, no linear relation between the strain and the MSD is found [12,20-22,30,31]. Thus, different relations between macroscopic strain and microscopic dynamics are observed after a constant stress or strain rate is applied. This indicates qualitative differences in otherwise analogous rheological protocols. Here, we compare the rheological responses to stress- and strain-controlled shear, respectively, in the transient and steady state of flow.

We investigate binary colloidal glasses with a large size, and hence dynamical, asymmetry. Depending on the number density and size ratio, different glass states exist in this system. At moderate size ratios, double glasses are found, in which both species are arrested. At larger size ratios and small to intermediate relative volume fractions of small spheres, single glasses are observed, in which the small particles are mobile in a glass of large particles. At large size ratios and large relative volume fractions of small spheres asymmetric glasses exist, in which the large particles are trapped in a glass of small spheres. The transitions between these glass states are accompanied by softening and melting of the glasses [31-33]. Due to the different nature of the glasses and the different involved arrest mechanisms, we expect different yielding mechanisms to occur. Therefore, using a single model system, a binary colloidal mixture, we can systematically study and compare the transitions from dynamical arrest to flow in different glass states.

\section{MATERIALS AND METHODS}

\section{A. Sample}

The samples contained sterically stabilized polymethylmethacrylate (PMMA) spheres with different radii: In the first system $R_{\mathrm{L} 1}=304 \mathrm{~nm} \quad(10 \%$ polydispersity $)$ and $R_{\mathrm{S} 1}=63 \mathrm{~nm}$ (15\% polydispersity) resulting in a size ratio $\delta=R_{\mathrm{S} 1} / R_{\mathrm{L} 1}=0.21$ and in the second system $R_{\mathrm{L} 2}=358 \mathrm{~nm}$ (14\% polydispersity) and $R_{\mathrm{S} 2}=137 \mathrm{~nm}$ (12\% polydispersity) resulting in $\delta=0.38$. The radii and polydispersities were determined using static and dynamic light scattering in very dilute samples with volume fractions $\phi<10^{-3}$. In the first system with $\delta=0.21$, the particles were suspended in a mixture of cis-decalin and cycloheptyl-bromide (CHB) which matches the density and almost the refractive index of the particles. In this mixture, PMMA colloids acquire a charge which was screened by adding salt, $4 \mathrm{mM}$ tetrabutylammoniumchloride [34]. For the second system with $\delta=0.38$, the particles were suspended in a mixture of octadecene and bromonaphtalene to minimize solvent evaporation [35]. In both solvent mixtures, the PMMA particles show hard-sphere-like behavior [36].
The volume fractions of sediments, obtained by centrifuging dilute suspensions, were estimated to be $\phi=0.67$ and 0.68 depending on the polydispersity [37]. One-component samples with a nominal volume fraction $\phi=0.61$ were obtained by diluting the sediments. Due to the uncertainties in the volume fractions, the volume fractions of the two associated suspensions were adjusted according to their normalized linear viscoelastic moduli, taking into account the trivial size dependence of the rheological response. For each value of $\delta$, one component was chosen as reference and the volume fraction of the suspension containing the second component are adjusted such that their normalized linear viscoelastic moduli are comparable. This procedure has been applied and described in detail previously [32]. By mixing the one-component stock suspensions, binary mixtures were obtained which had a total volume fraction $\phi=0.61$ and different compositions, i.e., fractions of small particles $x_{\mathrm{s}}=\phi_{\mathrm{s}} /$ $\phi$, where $\phi_{\mathrm{s}}$ is the volume fraction of the small component.

\section{B. Rheology}

For samples with $\delta=0.21$, creep measurements were performed using an AR2000ex stress-controlled rheometer (TA instruments) and a cone and plate geometry with diameter $D=20 \mathrm{~mm}$, cone angle $\alpha=2^{\circ}$ and truncation gap $d=0.054 \mathrm{~mm}$. For step-rate measurements, we used an ARES G2 strain-controlled rheometer (TA instruments) and cone and plate geometries with $D=25 \mathrm{~mm}, \alpha=2^{\circ}$, $d=0.048 \mathrm{~mm}$ and $D=50 \mathrm{~mm}, \alpha=1.16^{\circ}, d=0.051 \mathrm{~mm}$, respectively. Creep and step rate measurements of samples with $\delta=0.38$ were performed using an MCR 501 stress controlled rheometer (Anton Paar) with a cone and plate geometry with $D=25 \mathrm{~mm}, \alpha=3.22^{\circ}$, and $d=0.024 \mathrm{~mm}$. To minimize solvent evaporation, solvent traps were used which enclosed the samples in a small volume saturated with solvent vapor. To check whether the sample is affected by evaporation or other effects, the linear viscoelasticity was determined between the individual nonlinear measurements. If the measured viscoelasticities were deviated more than $1 \%-19 \%$ (depending on the noise in the individual measurements), the measurement series was stopped and a new sample was loaded.

A rejuvenation procedure was performed before each measurement to reduce the effects of loading, aging, and sample history and hence prepare each sample in a reproducible initial state. To achieve this, directly after loading first a dynamical strain sweep (DSS), i.e., oscillatory shear with a frequency $\omega=1 \mathrm{rad} / \mathrm{s}$ and an increasing strain from $\gamma=0.002 \%$ to between $500 \%$ and $1000 \%$ (depending on the sample), was performed in order to determine the flow regime. The onset of flow was determined from the onset of the terminal relaxation of the moduli, characterized by power-law dependencies of the moduli on strain amplitude [38]. Subsequently and before each measurement, two dynamical time sweeps (DTS) were applied. For $100 \mathrm{~s}$, a time sufficient to reach steady state values of the moduli in all samples, a DTS was performed with a strain in the flow regime, $\gamma \geq 300 \%$ (as determined from the previous DSS), to fluidize the sample and thus remove loading, aging, and 
history effects. Subsequently, a DTS was performed in the linear viscoelastic regime, $\gamma=0.003 \%-0.1 \%$ (depending on the sample), and extended until the elastic, $G^{\prime}$, and viscous, $G^{\prime \prime}$, moduli reached constant, steady state values. The time needed to achieve a steady state also depends on the sample. This procedure ensures that each sample is in a reproducible state before they are subjected to a rheological test.

\section{RESULTS AND DISCUSSION}

\section{A. Steady-state flow curve}

The response in a steady-state shear flow is characterized by the flow curve, in which the shear stress, $\sigma$, is plotted as a function of the applied shear rate $\dot{\gamma}$ (Fig. 1, inset). Data obtained upon increasing and decreasing the shear rate, respectively, show no significant difference (Fig. 1, inset, continuous and dashed lines, respectively).

In order to account for trivial effects due to the different average particle sizes, the stress is also reported in units of the energy density $k_{\mathrm{B}} T /\left\langle R^{3}\right\rangle=k_{\mathrm{B}} T\left[1-x_{\mathrm{s}}\left(1-1 / \delta^{3}\right)\right] / R_{\mathrm{L}}^{3}$ and plotted as a function of the Peclet number $P e=\dot{\gamma}\left\langle\tau^{\mathrm{s}}\right\rangle=$ $\dot{\gamma}\left\langle\tau_{0}^{\mathrm{s}}\right\rangle / f$ with $\left\langle\tau_{0}^{\mathrm{s}}\right\rangle=6 \pi \eta\left\langle R^{3}\right\rangle / k_{\mathrm{B}} T$ the average short-time Brownian time in the dilute limit. The factor $f$ is estimated to be $f \approx 1 / 32$ for $\phi=0.61$, obtained by extrapolating the data in Fig. 8 of [39]. Note that the short-time Brownian time in the dilute limit, $\left\langle\tau_{0}^{\mathrm{s}}\right\rangle$, and hence $P e$ does not reflect the softening of the glasses discussed below.

The flow curves reveal two distinct ranges of $P e$. At large $P e$, for all samples a sublinear increase of the stress $\sigma$ with increasing $P e$ is observed. It indicates shear thinning with the viscosity decreasing with shear rate $\dot{\gamma}[11,13,19,21,22,30,40,41]$. At low $P e$ two distinct behaviors are observed. For glassy systems, in the limit of $P e \rightarrow 0$ the stress tends toward a constant value, the yield stress. Its value cannot be determined

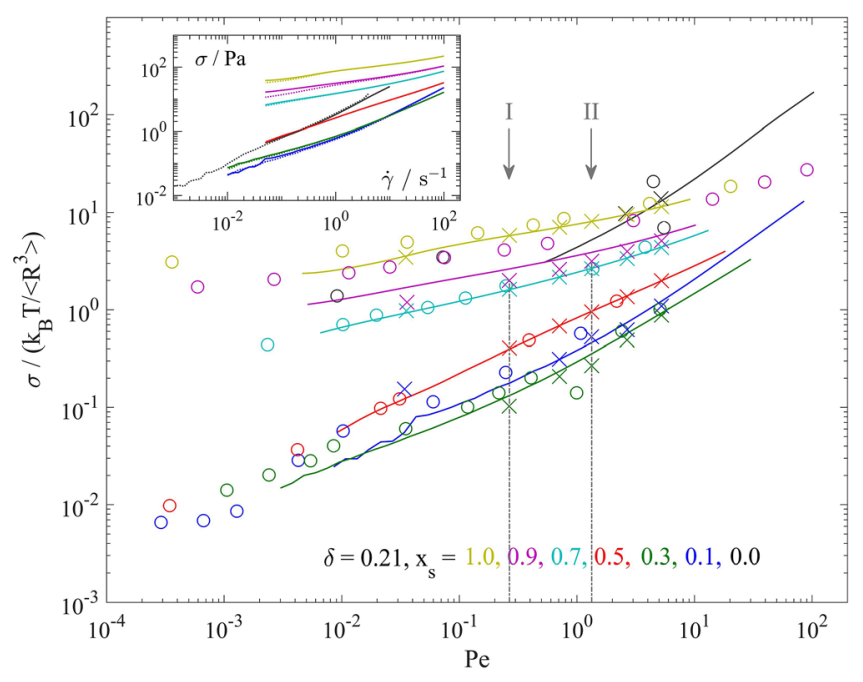

FIG. 1. Flow curves, that is stress $\sigma$ in units of the energy density $k_{\mathrm{B}} T /\left\langle R^{3}\right\rangle$ as a function of shear rate $\dot{\gamma}$ in units of the Brownian time, that is Peclet number $P e$ (in the inset the data are not normalized), measured by increasing (continuous lines) or decreasing (dashed lines) the shear rate $\dot{\gamma}$ as well as extracted from step rate experiments (crosses) and step stress, i.e., creep, experiments (circles). The conditions shown in Fig. 8 are indicated by arrows and dashed-dotted lines. Samples with size ratio $\delta=0.21$ and different compositions $x_{\mathrm{s}}$ (as indicated). precisely due to the limited accessible range of $P e$. However, an extrapolation to low $P e$ suggests values between 1 and $10 k_{\mathrm{B}} T /\left\langle R^{3}\right\rangle$. This range compares well with previous studies on hard sphere glasses [10,42] and is lower than those obtained for soft spheres $[42,43]$. This is observed for samples with $x_{\mathrm{s}}=0.7,0.9$, and 1.0. In contrast, a stress that decreases with decreasing $P e$ also for the smallest investigated $P e$ indicates fluid samples, here with intermediate compositions $x_{\mathrm{s}}=0.1,0.3$, and 0.5 . For the sample with $x_{\mathrm{s}}=0.0$, the range of explored $P e$ values does not extend to sufficiently low $P e$ to reveal the existence of a yield stress. However, there is no reason to assume that this sample does not have a yield stress.

The one-component systems, $x_{\mathrm{s}}=0$ and $x_{\mathrm{s}}=1$, represent the same glass state and correspondingly possess similar values of the stress in dimensionless units. Nevertheless, the shapes of the flow curves show differences, especially at the largest studied $P e$. For $x_{\mathrm{s}}=0$, the slope is about 0.85, whereas for $x_{\mathrm{s}}=1$ it is significantly smaller, about 0.3 . However, these slopes are not expected to be the asymptotic slopes as the range of studied $P e$ is limited. Slopes between 0.5 and 0.75 were observed in previous studies on similar hard sphere systems $[13,38]$. Other soft glassy materials, such as dispersions of soft spheres, emulsions, or foams, typically exhibit slopes between 0.5 and $0.6[38,44,45]$. The smaller slope of the one-component system of small particles may therefore be due to the larger polydispersity and a more pronounced softness of the small particles. The latter might be due to the different extent of the stabilizing polymer layer relative to the particle radius; about $7.5 \%$ for the small particles compared to about $1.5 \%$ for the large particles. The slopes of the flow curves for intermediate mixing ratios appear to be interpolations between the values obtained for $x_{\mathrm{s}}=0$ and $x_{\mathrm{s}}=1$, which might reflect the weighting of the two softnesses and polydispersities.

Adding small spheres to a glass of large spheres, i.e., increasing $x_{\mathrm{s}}$, for all $\mathrm{Pe}$ the normalized stress decreases and reaches a minimum at $x_{\mathrm{s}}=0.3$ before it increases again. Moreover, for intermediate $x_{\mathrm{s}}$ the stress plateau at low rates disappears and the stress continuously decreases with decreasing $P e$. Both observations indicate the softening for intermediate $x_{\mathrm{s}}$ and a subsequent reentrant vitrification. This agrees with previous experimental results [46], including DSS tests [32] and step-rate experiments [31], and theoretical calculations [47,48]. It is thought to reflect the transition from a glass in which the cage is formed by large spheres at small $x_{\mathrm{s}}$, to a glass where the cage is formed by small spheres at large $x_{\mathrm{s}}[31,32]$.

When the flow curve is determined, the shear rate is successively increased to measure the individual data points. We also obtained data upon decreasing the shear rate, which show no significant difference (Fig. 1, continuous and dashed lines, respectively). Nevertheless, with both protocols for each data point the initial state of the sample is the steadystate of flow reached by the application of the previous $\dot{\gamma}$. For comparison, $\sigma(\dot{\gamma})$ is also extracted from a series of steprate experiments. In this case, after rejuvenation the initially quiescent sample is subjected to a constant shear rate $\dot{\gamma}$ and the stress $\sigma$ as a function of strain $\gamma$ measured. The constant 
steady-state stress is reached at large $\gamma$ and also reported in Fig. 1 (crosses). All protocols result in comparable $\sigma(\dot{\gamma})$, indicating that the flow curve measurement represents steady-state stress values. Another way to measure the rate dependent stress $\sigma(\dot{\gamma})$ is provided through a series of creep experiments, where a constant stress $\sigma$ is applied and the strain $\gamma$ or strain rate $\dot{\gamma}$ is measured as a function of time $t$. In the creep regime, i.e., below the yield stress $\sigma \lesssim \sigma_{y}$, the strain rate slowly decreases with time without reaching a constant value and hence no steady state of flow is reached. In contrast, for stresses beyond the yield stress, $\sigma>\sigma_{\mathrm{y}}$, the shear rate $\dot{\gamma}$ reaches a steady-state value at long times. These values (Fig. 1, full circles) agree with the flow curve or are slightly larger. The agreement between steady-state stress values obtained with different shear protocols as well as for different one-component and binary systems in different fluid and glass states, i.e., with different compositions $x_{\mathrm{s}}$, indicates that, for all these systems, a comparable steady state of flow is reached independent of the shear protocol [49]. Thus, steps to either a constant shear rate $\dot{\gamma}$ or a constant stress $\sigma>\sigma_{\mathrm{y}}$ result in the same $\sigma(\dot{\gamma})$ as classical flow curve measurements.

\section{B. Step-stress experiments (creep)}

In creep experiments, a constant shear stress $\sigma$ is applied and the induced deformation $\gamma$ or deformation rate $\dot{\gamma}$ is recorded as a function of time $t$. This is illustrated for the one-component glass [Fig. 2(a)]. The response significantly depends on whether a stress above or below the yield stress $\sigma_{\mathrm{y}}$ is applied. The yield stress $\sigma_{\mathrm{y}}$ was estimated from the crossing point of the shear moduli $G^{\prime}$ and $G^{\prime \prime}$ measured in DSS with an angular frequency $\omega=1 \mathrm{rad} / \mathrm{s}$. For this sample $\left(R_{\mathrm{S} 1}, x_{\mathrm{s}}=1.0\right)$, we determined $\sigma_{\mathrm{y}} \approx 80 \quad$ Pa. At short times, the initial superlinear increase of the strain $\gamma(t)$ is followed by oscillations which are caused by instrument inertia [50] and will not be discussed further. After this initial stage, for stresses below the yield stress, $\sigma<\sigma_{\mathrm{y}}$, a creep response is observed at sufficiently long times. The creep response is characterized by a slow sublinear increase of strain which can be separated into two regimes: At intermediate times (immediately after the regime affected by instrument inertia) an extremely slow increase of $\gamma(t)$ and at long times a stronger but still sublinear increase of $\gamma(t)$ is observed. This long-time regime is in general observed for yield-stress fluids and is known as Andrade creep for a variety of systems $[15,28,44,51]$. Recently, it was shown that these two regimes are characteristic for creep in colloidal glasses $[14,15,50]$ and colloidal gels [52,53]. For larger applied stresses, $\sigma>\sigma_{\mathrm{y}}$, the system flows and a linear time dependence of the strain $\gamma(t)$ is observed. If a stress close to the yield stress is applied, $\sigma \approx \sigma_{\mathrm{y}}$, an intermediate behavior is found. The creeplike response at intermediate times is followed by a superlinear increase of $\gamma(t)$ over a limited time interval, after which a steady state of flow is reached. The superlinear increase of $\gamma(t)$ implies a progressively decreasing instantaneous viscosity and hence shear thinning, while flow is associated with a constant viscosity.
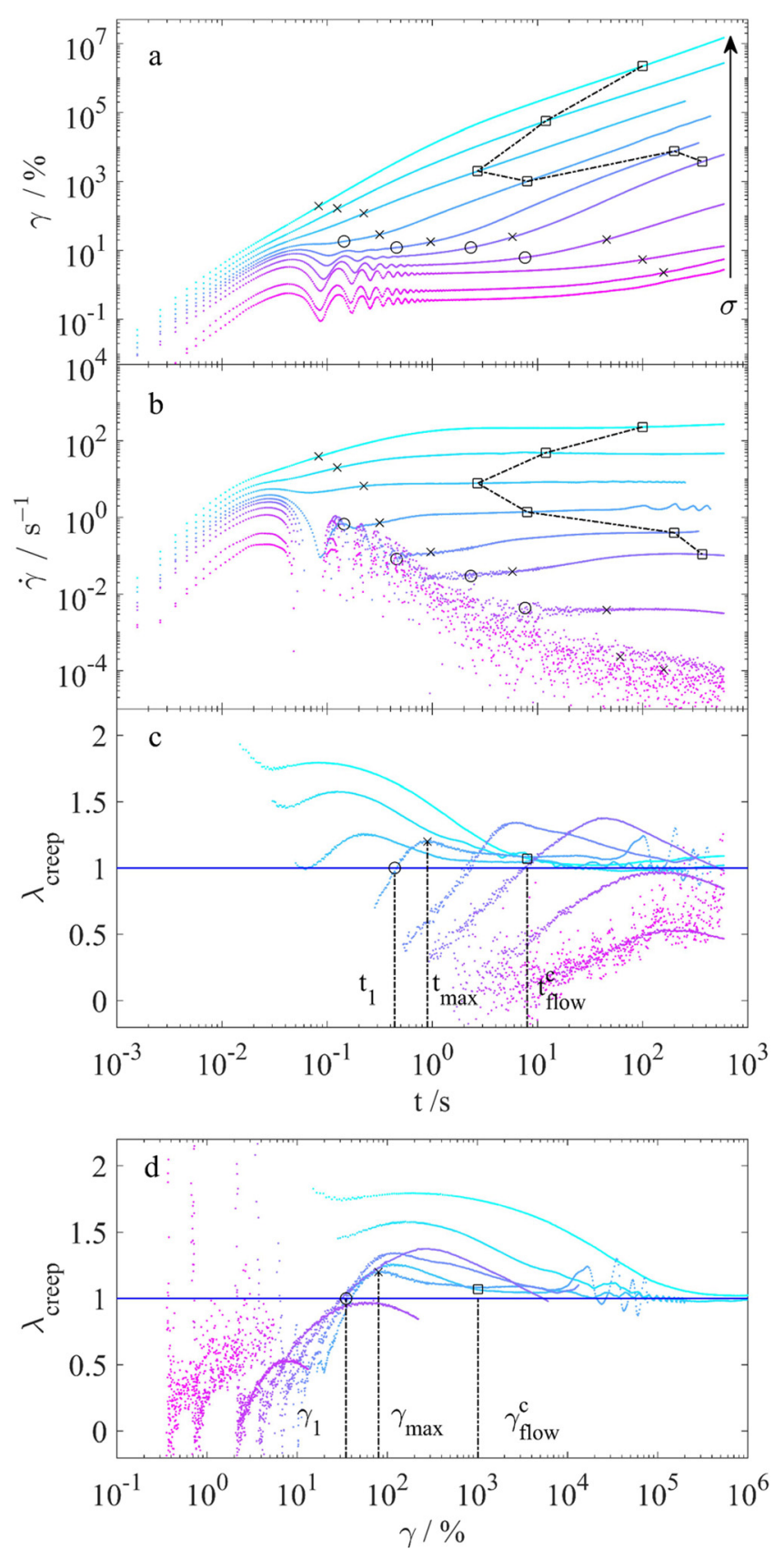

FIG. 2. (a) Strain $\gamma$ and (b) shear rate $\dot{\gamma}$ as a function of time $t$ measured in step stress (creep) experiments with stresses $\sigma=10,15,35,50,65,80,100$, 140,200 , and $300 \mathrm{~Pa}$ (from bottom to top). (c) and (d) Logarithmic time derivative of the strain $\lambda_{\text {creep }}(t)=\mathrm{d} \log (\gamma) / \mathrm{d} \log (t)$ as a function of (c) time $t$ and (d) strain $\gamma$. Time $t_{1}$ and strain $\gamma_{1}(\mathrm{O})$ are defined by $\lambda_{\text {creep }}=1$, time $t_{\max }$ and strain $\gamma_{\text {max }}(\times)$ indicate the maximum of $\lambda_{\text {creep }}$, and time $t_{\text {flow }}^{\mathrm{c}}$ and $\gamma_{\text {flow }}^{\mathrm{c}}$ ( $\square$ ) indicate when the steady state of flow is reached. Samples contain only particles of radius $R_{\mathrm{S} 1}$, i.e., $x_{\mathrm{s}}=1.0$.

Recent studies indicate that inhomogeneous flow might occur during the transition to steady flow [24,54-56].

For one-component and binary glasses, it has been shown that the superlinear increase of $\gamma(t)$ can be associated with super-diffusive dynamics and the opening of shear-deformed cages which ultimately leads to diffusion [14,24]. While the superlinear behavior is well-developed for the smallest PMMA particles used in this study (Fig. 2), it is less clear for the other single component samples investigated here and samples studied previously [15]. This might be due to the 
softness of the smaller PMMA particles which is expected to promote the superlinear behavior [57].

The creep response of the binary mixtures is shown for normalized stresses $\sigma /\left(k_{\mathrm{B}} T /\left\langle R^{3}\right\rangle\right)$ (Fig. 3). Depending on the composition $x_{\mathrm{s}}$, different scenarios are observed. For the one-component glasses, the applied stress is smaller than the yield stress and correspondingly a creeplike response is observed. The yield stress of the one-component glasses is about $1 k_{\mathrm{B}} T /\left\langle R^{3}\right\rangle$, in agreement with previous investigations on hard-sphere glasses [10,42]. In contrast, a fluidlike response is found for mixtures with intermediate $x_{\mathrm{s}}$. This effect is not symmetric with respect to the composition: For $\delta=0.21$ the strongest softening occurs at $x_{\mathrm{s}} \approx 0.3$ [Fig. 3(a)] $[31,33]$, whereas for $\delta=0.38$ the sample with $x_{\mathrm{s}}=0.25$ still shows a creep response, in agreement with linear viscoelasticity measurements [32]. With decreasing $\delta$ hence the maximum softening seems to move toward smaller $x_{\mathrm{s}}$, i.e., the softening becomes more asymmetric with respect to $x_{\mathrm{s}}$. Moreover, with decreasing $\delta$ the softening becomes more pronounced; for $\delta=0.21$ flow is reached almost immediately at low and intermediate compositions $x_{\mathrm{s}}$. An intermediate behavior, with a transition from creep to flow, was observed for $\delta=0.21$ and $x_{\mathrm{s}}=0.7$ as well as for $\delta=0.38$ and $x_{\mathrm{s}}=0.5$ and 0.75 . This is consistent with softening at intermediate $x_{\mathrm{s}}$ as well as with the flow curves (Fig. 1) and other studies [31-33].

As mentioned above, the evolution of $\gamma(t)$ shows different regimes characterized by differently increasing $\gamma(t)$. To quantify the transitions between these regimes, the logarithmic time derivative of the strain $\lambda_{\text {creep }}(t)=\mathrm{d} \log (\gamma) / \mathrm{d} \log (t)$ is calculated. This implies $\gamma \sim t^{\lambda_{\text {creep }}}$ and hence a comparison of $\lambda_{\text {creep }}(t)$ with 1 identifies sublinear, linear and superlinear

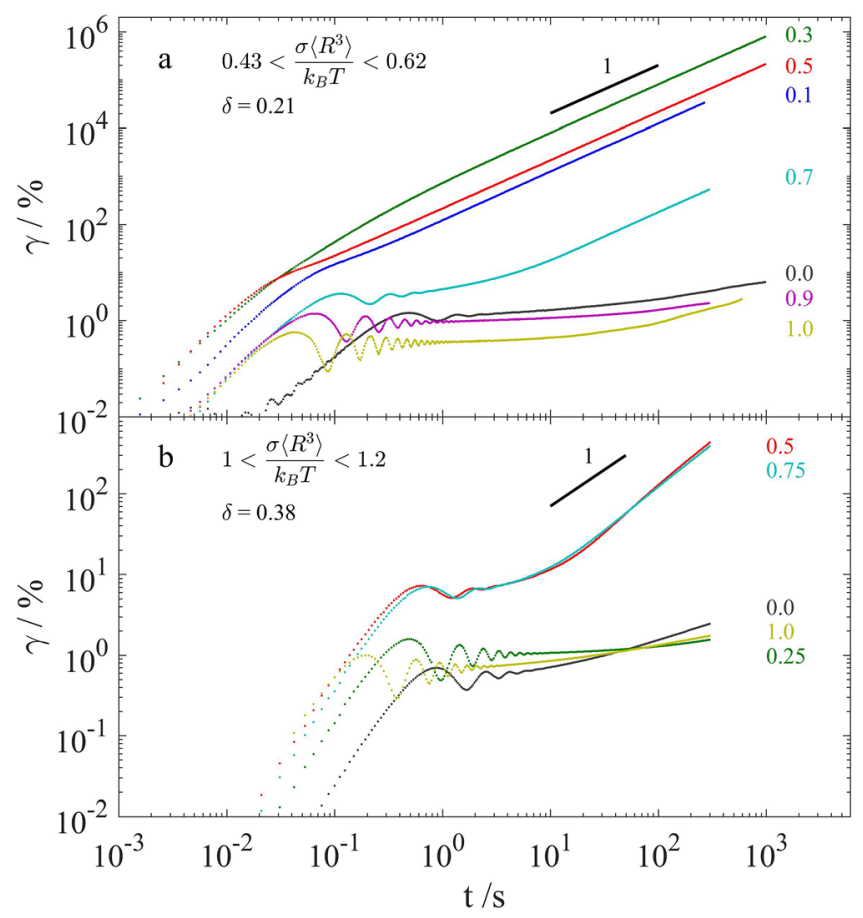

FIG. 3. Strain $\gamma$ as a function of time $t$ measured in creep experiments performed on samples with size ratio (a) $\delta=0.21$ and (b) $\delta=0.38$ and composition $x_{\mathrm{s}}$ (as indicated) at similar normalized stresses $\sigma /\left(k_{\mathrm{B}} T /\left\langle R^{3}\right\rangle\right)$. dependencies of $\gamma$ on $t$. Figures 2(c) and 2(d) show $\lambda_{\text {creep }}(t)$ for the one-component glass of small spheres as a function of $t$ and $\gamma$, respectively. Due to instrument inertia, the initial regime is characterized by a fast decrease from an initial exponent $\lambda_{\text {creep }}=2$ and by oscillations. Subsequently, for $\sigma>\sigma_{\mathrm{y}}$, the value of $\lambda_{\text {creep }}(t)$ starts to increase from below 1 , reaches a maximum larger than 1 and finally tends to 1 , which characterizes flow. Deviations from this behavior are observed for $\sigma<\sigma_{\mathrm{y}}$, where the maximum value is smaller than 1 , and for $\sigma \gg \sigma_{\mathrm{y}}$, where no clear maximum is observed.

Based on $\lambda_{\text {creep }}(t)$ and the corresponding $\lambda_{\text {creep }}(\gamma)$ [Figs. 2(c) and 2(d)], we can determine the transitions and extract the corresponding times and strains. The requirement $\lambda_{\text {creep }}\left(t_{1}\right)=1$ defines $t_{1}$ and $\gamma_{1}$, which marks the transition from the creep regime, i.e., $\lambda_{\text {creep }}<1$, to the superlinear regime, i.e., $\lambda_{\text {creep }}>1$. Furthermore, the maximum of $\lambda_{\text {creep }}(t)$ occurs at $t_{\max }$ and $\gamma_{\max }$. The final steady-state flow regime is reached once $\lambda_{\text {creep }}(t)$ approaches 1 , i.e., $\gamma$ depends linearly on $t$, which occurs beyond about $t_{\text {flow }}^{\mathrm{c}}$ and $\gamma_{\text {flow }}^{\mathrm{c}}$.

The strains $\gamma_{1}$ and $\gamma_{\max }$ increase with increasing stress $\sigma$ [Figs. 4(b) and 4(c)]. We find a scaling $\gamma_{\max } \sim \sigma^{\alpha}$ with $\alpha=0.3$, independent of the size ratio $\delta$ and composition $x_{\mathrm{s}}$, while the dependence of $\gamma_{1}$ on $\sigma$ is weaker and less clear. The increase of $\gamma_{1}$ and $\gamma_{\max }$ can be understood in terms of the balance between Brownian motion and shear-induced motion together with the cage effect. With increasing stress $\sigma$, Brownian motion becomes less important and affine motions as well as shear-induced diffusion due to particle collisions

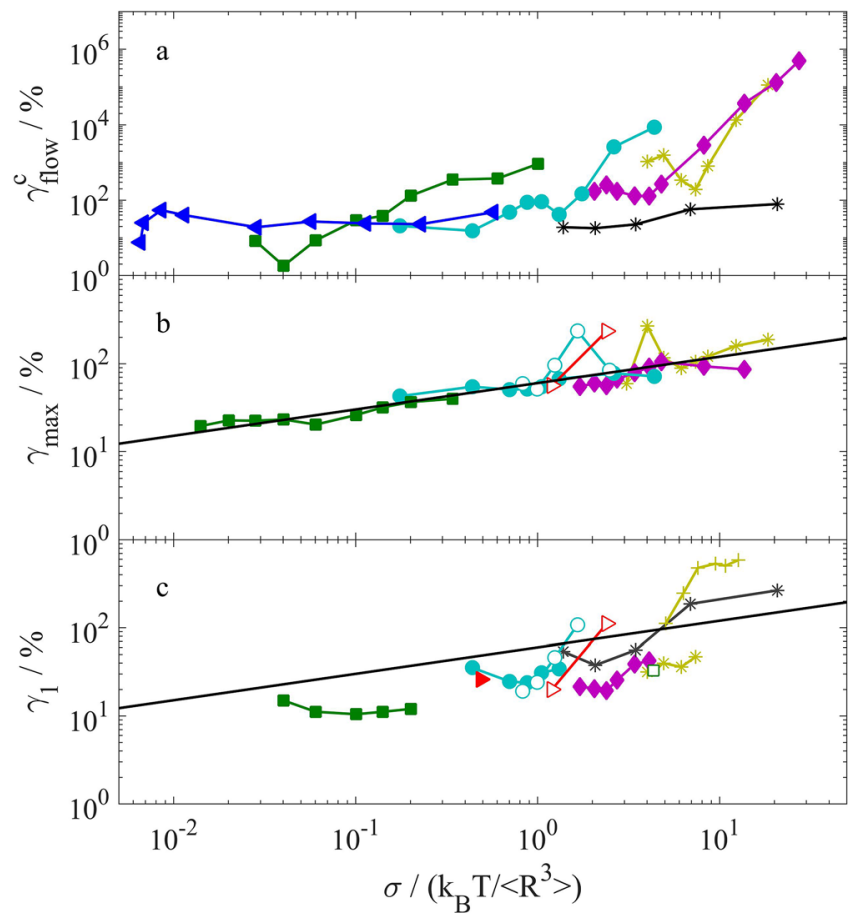

FIG. 4. (a) Strain $\gamma_{\text {flow }}^{\text {c }}$ required to reach the steady state of flow, i.e., where $\lambda_{\text {creep }}$ becomes about 1 , (b) strain $\gamma_{\max }$ where $\lambda_{\text {creep }}$ reaches its maximum and (c) strain $\gamma_{1}$ where $\lambda_{\text {creep }}$ crosses 1 for the first time as a function of normalized stress $\sigma /\left(k_{\mathrm{B}} T /\left\langle R^{3}\right\rangle\right)$ for samples with $\delta=0.21$ and compositions $x_{\mathrm{s}}=0.0(*), 0.1(\varangle), 0.3(\square), 0.5(\bullet), 0.7(\bullet), 0.9(\bullet), 1.0\left(^{*}\right)$ as well as $\delta=0.38$ and $x_{\mathrm{s}}=0.25(\square), 0.5(\triangleright), 0.75(\mathrm{O}), 1.0(+)$. The black lines represent fits with $\gamma \sim \sigma^{\alpha}$. 
start to dominate. Thus, at large $\sigma \dot{\gamma}$ the cage is deformed more before it breaks due to shear-induced particle collisions. Therefore, $\gamma_{1}$ and $\gamma_{\max }$ increase as $\sigma$ increases.

The strain $\gamma_{\text {flow }}^{\text {c }}$ that is needed to reach steady-state flow shows two regimes upon increasing $\sigma$. At low applied stresses, $\gamma_{\text {flow }}^{\mathrm{c}}$ is almost constant for all $x_{\mathrm{s}}$, whereas it starts to increase at large $\sigma$. Similar to the above argument, the increase at large $\sigma$ suggests that, for these $\sigma$, cage deformations become controlled by shear [12,19,31]. For the different $x_{\mathrm{s}}$, the transition occurs at different $\sigma$, which is attributed to the dependence of the modulus on the state of the sample, i.e., its composition $x_{\mathrm{s}}$.

\section{Step-rate experiments}

In a step-rate experiment, the evolution of the stress $\sigma$ is measured as a function of strain $\gamma$ (or time $t=\gamma / \dot{\gamma}$ ) under application of a constant shear rate $\dot{\gamma}$. Typically, $\sigma(\gamma)$ shows an initial linear increase, deviations from linear behavior followed by a maximum, the stress overshoot, and finally a constant stress corresponding to the steady state of flow (Fig. 5). The stress overshoot was found to be related to the maximum cage deformation before the cage breaks [19,20,58]. Moreover, the singe-particle dynamics reveal a transient superdiffusive regime at deformations that are comparable to those of the stress overshoot [12,19-22]. In particular at low shear rates, shear inhomogeneities may be present $[59,60]$.

For one-component samples, the overshoot occurs at increasing strain $\gamma_{\text {peak }}$ and becomes less pronounced as the shear rate $\dot{\gamma}$ or Peclet number $P e$ increase (Fig. 5). For the same $P e$, the two one-component systems show a similar response, if the normalized stress $\sigma /\left(k_{\mathrm{B}} T /\left\langle R^{3}\right\rangle\right)$ is considered [Fig. 6(b)]. The smaller particles show a slightly more pronounced overshoot, which is attributed to their slightly softer interactions and is consistent with the observations in the flow curve and creep experiments (Figs. 1 and 3 ) and previous findings [38]. In contrast, a pronounced reduction of

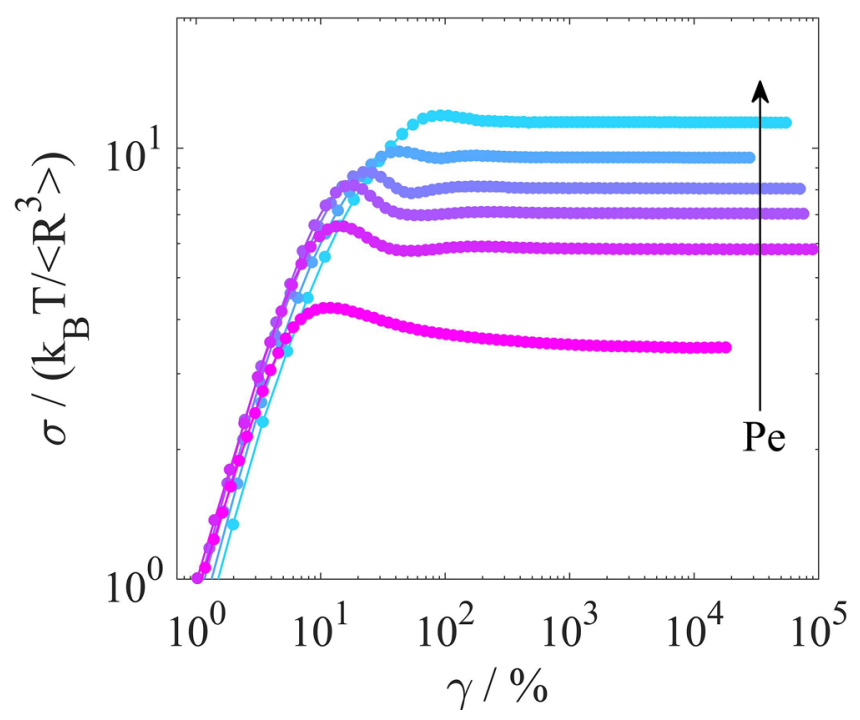

FIG. 5. Normalized stress $\sigma /\left(k_{\mathrm{B}} T /\left\langle R^{3}\right\rangle\right)$ as a function of strain $\gamma$ measured in step-rate experiments with shear rates corresponding to Peclet numbers $P e=0.032,0.24,0.64,1.2,2.4$, and 4.7 (from bottom to top). Samples contain only particles of radius $R_{\mathrm{S} 1}$, i.e., $x_{\mathrm{s}}=1.0$.

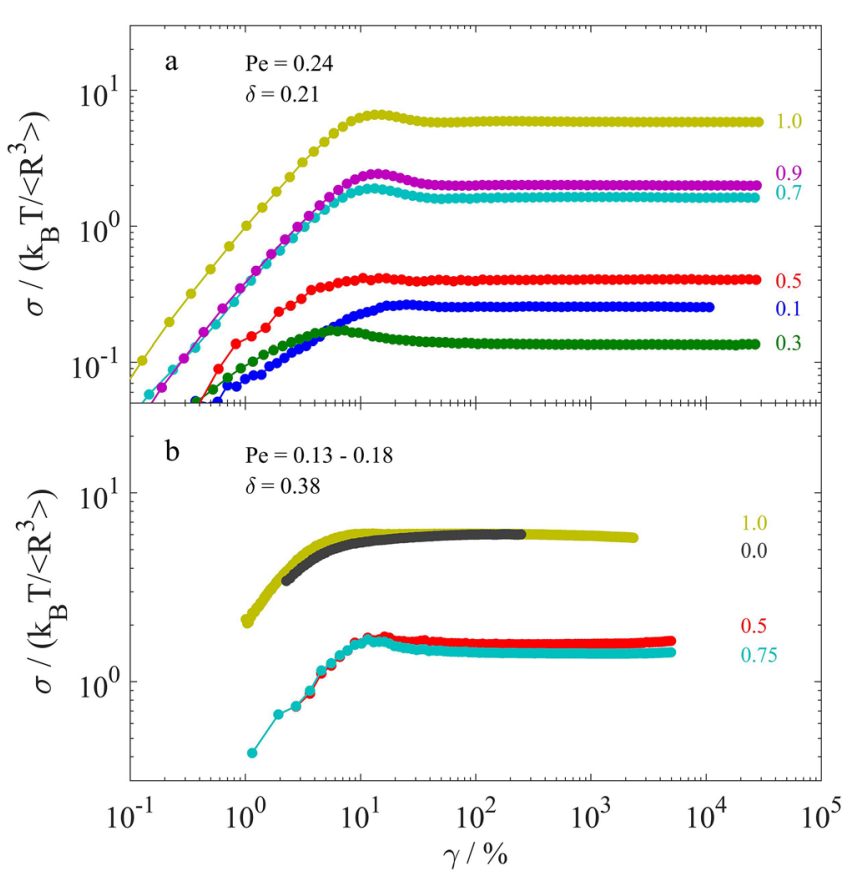

FIG. 6. Normalized stress $\sigma /\left(k_{\mathrm{B}} T /\left\langle R^{3}\right\rangle\right)$ as a function of strain $\gamma$ measured in step-rate experiments with shear rates corresponding to (a) Peclet number $P e=0.24$ for samples with size ratio $\delta=0.21$ and composition $x_{\mathrm{s}}$ (as indicated) and (b) $P e=0.13-0.18$ for $\delta=0.38$ and $x_{\mathrm{s}}$ as indicated.

the stress is observed at intermediate $x_{\mathrm{s}}$ with the effect being more pronounced for the smaller size ratio, $\delta=0.21$ (Fig. 6). This is consistent with the observations in the creep experiments (Fig. 3) and the yield stress previously measured in DSS [32]. It is attributed to the softening effect discussed above (Sec. III B).

To quantify the dependence on the composition $x_{\mathrm{s}}$ and shear rate $\dot{\gamma}$ or Peclet number $P e$, the strain at the overshoot, $\gamma_{\text {peak, }}$ the magnitude of the overshoot, $\sigma_{\text {peak }} / \sigma_{\text {steady }}-1$, and the strain when steady-state flow is approximately reached, $\gamma_{\text {flow }}^{\text {sr }}$, are extracted from the measurements as a function of composition $x_{\mathrm{s}}$ and $\mathrm{Pe}$ (Fig. 7). The steady state of flow is assumed to be reached when the stress becomes approximately constant, quantified by a condition for the logarithmic time derivative of the stress $\mathrm{d} \log (\sigma) / \mathrm{d} \log (t)<0.1$. For both size ratios $\delta$ and all compositions $x_{\mathrm{s}}$, the strain at the overshoot $\gamma_{\text {peak }}$ changes only little for $P e<1$. In this Peclet number range, Brownian motion dominates over the influence of shear and thus the shear-induced cage deformation is not important. Therefore, the escape from cages is mainly due to Brownian motion and $\gamma_{\text {peak }}$ is essentially independent of $\dot{\gamma}$. For $P e>1$, however, $\gamma_{\text {peak }}$ is found to increase. Under these conditions, the particle motion is increasingly dominated by affine and shear-induced diffusive motions, in agreement with previous work [12,22,31] and analogous to the evolution in creep experiments (Fig. 4). A similar dependence is observed for the strain $\gamma_{\text {flow }}^{\text {sr }}$ and the corresponding time $t_{\text {flow }}^{\text {sr }}=\gamma_{\text {flow }}^{\text {sr }} / \dot{\gamma}$ at which the steady state of flow is approximately reached [Fig. 7(a)]. In this case, the data show a larger spread which we attribute to the slow approach to the steady state and hence the not very well defined transition to steady-state flow.

The magnitude of the stress overshoot, $\sigma_{\text {peak }} / \sigma_{\text {steady }}-1$ [Fig. 7(c)], characterizes the ability to store and release 


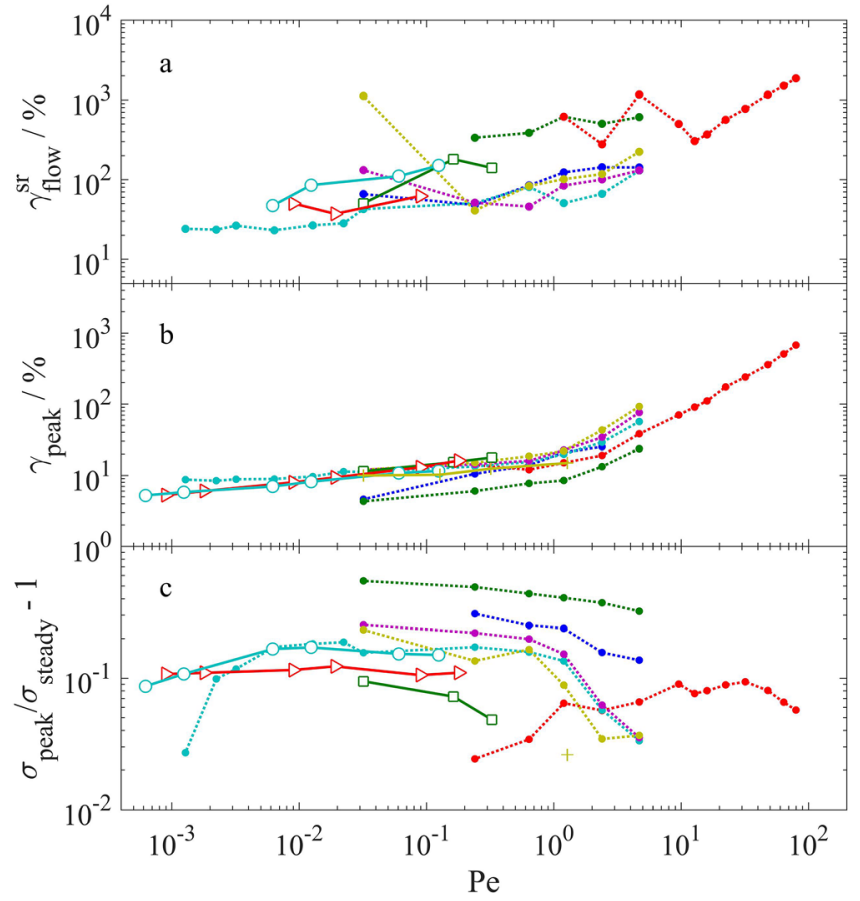

FIG. 7. (a) Strain when the steady state of flow is approximately reached, $\gamma_{\text {flow }}^{\mathrm{sr}}$, (b) strain at the stress overshoot, $\gamma_{\text {peak }}$, and (c) magnitude of the stress overshoot, $\sigma_{\text {peak }} / \sigma_{\text {steady }}-1$, as a function of Peclet number $P e$ for size ratio $\delta=0.21$ and composition $x_{\mathrm{s}}=0.1(\bullet), 0.3(\bullet), 0.5(\bullet), 0.7(\bullet), 0.9(\bullet)$, $1.0(\bullet)$ [31] and for $\delta=0.38$ and $x_{\mathrm{s}}=0.25(\square), 0.5(\triangleright), 0.75(\mathrm{O}), 1.0(+)$.

stress. As a function of $P e$, it shows a nonmonotonic behavior with a maximum at intermediate $P e$, whose position depends on the specific sample. This is observed for samples with $\delta=0.21$ and intermediate compositions $x_{\mathrm{s}}=0.5$ and 0.7 , whereas for the other samples the stress overshoot at small $P e$ is too small to be quantified unambiguously. This nonmonotonic behavior, as in the creep experiments, has been associated with the competition between the timescales of the structural relaxation of the caging species, which dominates at small $\dot{\gamma}$, and of shear, which dominates at high $\dot{\gamma}[12,19,31]$. The maximum reflects the transition between both regimes and hence depends on the balance between the structural relaxation time and the imposed time scale $1 / \dot{\gamma}$. The structural relaxation time depends on the composition $x_{\mathrm{s}}$. Hence the transition occurs at smaller $P e$ in the glassy samples and at higher $P e$ in the fluid samples, i.e., intermediate $x_{\mathrm{s}}$. The nonmonotonic behavior is more pronounced for $\delta=0.21$. These trends are consistent with the results of the creep experiments. It is related to the degree of cage compressibility which similarly varies as a function of $x_{\mathrm{s}}$ and reflects the transition from a cage of large spheres to a cage of small spheres [31]. For $\delta=0.38$, the overshoot only increases slightly with increasing $x_{\mathrm{s}}$ [Fig. 7(c)]. This might be related to the absence of a well-defined cage transition for smaller size asymmetries.

\section{Comparison of step-stress and step-rate experiments}

Now we contrast the findings obtained in the creep experiments with those of the step-rate experiments. It was shown that they both lead to very similar steady states of flow (Fig. 1).
However, they follow different paths to the steady state of flow. To elucidate this, we compare the transient regimes.

Figure 8 shows two creep and two step-rate measurements for the sample with $\delta=0.21$ and $x_{\mathrm{s}}=0.7$. In a creep experiment, the steady flow is characterized by a linear increase of the strain with time, i.e., a regime of constant shear rate. In the two examples, the shear rates are $\dot{\gamma}(\sigma=20 \mathrm{~Pa}) \approx 1.9 \mathrm{~s}^{-1}$ (condition I) and $\dot{\gamma}(\sigma=30 \mathrm{~Pa}) \approx 10.4 \mathrm{~s}^{-1}$ (condition II) [Fig. $8(\mathrm{a})]$. On the other hand, in the step-rate experiments similar shear rates, $\dot{\gamma}=2.0 \mathrm{~s}^{-1}$ and $\dot{\gamma}=10.2 \mathrm{~s}^{-1}$, are applied [Fig. 8(b)] and in the steady-state of flow $\sigma \approx 19 \mathrm{~Pa}$ and $\sigma \approx 30 \mathrm{~Pa}$ are measured, which are similar to the stresses applied in the creep experiments. Both shear protocols, stress- and straincontrolled, lead to the fluidization of the glass and result in comparable steady states of flow with very similar stresses and strains (indicated in Fig. 1).

To compare the transient regimes, the data obtained following the two protocols are represented in a joint graph as the product $\sigma \dot{\gamma}(t)$ [Fig. 8(c)]. The data at long times indeed show that the steady state of flow is the same for the creep and step-rate experiments. In the transient regime, however, the responses differ and hence the sample follows a different path toward the steady state of flow under constant stress and constant shear rate, respectively. For the step-rate experiment, $\sigma \dot{\gamma}(t)$ resembles $\sigma(\gamma)$ since $\dot{\gamma}$ is constant and the strain $\gamma=\dot{\gamma}$ t. Initially, it steeply increases with time and, beyond the overshoot, decreases to a constant value. In the stresscontrolled (creep) case, however, the increase of $\sigma \dot{\gamma}$ is more gradual and hence steady flow is achieved later. While this difference is observed in both cases, it is more pronounced for the larger stress $\sigma=30 \mathrm{~Pa}$ and strain $\dot{\gamma}=10.2 \mathrm{~s}^{-1}$ (condition II). Note that these differences cannot result from thixotropic behavior, as for example observed in colloidal gels [51]. Our samples do not show any relevant bifurcation in the flow curves measured with increasing and decreasing shear rates (Fig. 1). In addition, the absence of two-step yielding in step rate experiments (Fig. 6), as well as previous results obtained under oscillatory shear [32] seem to indicate that attractive glasses or gels are not formed in the present mixtures [61].

To quantify these differences, based on $\gamma_{\text {flow }}^{\mathrm{c}}(\sigma)$ [Fig. 4(a)] and $\gamma_{\text {flow }}^{\text {sr }}(\dot{\gamma})\left[\right.$ Fig. 7(a)] the time $t_{\text {flow }}=\gamma_{\text {flow }} / \dot{\gamma}$ required to reach the steady state of flow in creep and step-rate experiments, respectively, is considered as a function of $\sigma \dot{\gamma}$, which allows for a direct comparison of the times $t_{\text {flow }}$ determined following both protocols [Fig. 9(a)]. In this comparison, the product $\sigma \dot{\gamma}$ is based on the steady-state value $\dot{\gamma}(t \rightarrow \infty)$ in the case of creep experiment where a constant stress $\sigma$ is applied and on the steady-state value $\sigma(\gamma \rightarrow \infty)$ in step-rate experiments where a constant shear rate $\dot{\gamma}$ is applied. In creep experiments, $t_{\text {flow }}$ is found to initially decrease but then to increase again with $\sigma \dot{\gamma}$, as indicated by the bend formed by the squares in Fig. 2(a). In step-rate experiments, however, $t_{\text {flow }}$ monotonically decreases with increasing $\sigma \dot{\gamma}$. For small $\sigma \dot{\gamma}, t_{\text {flow }}$ observed in step rate experiments are slightly smaller than those observed in creep experiments, but in general they show very similar behavior, namely, a decrease with increasing $\sigma \dot{\gamma}$. This regime of decreasing $t_{\text {flow }}$ corresponds to the approximately constant $\gamma_{\text {flow }}^{\mathrm{c}}(\sigma)$ [Fig. 4(a)], 

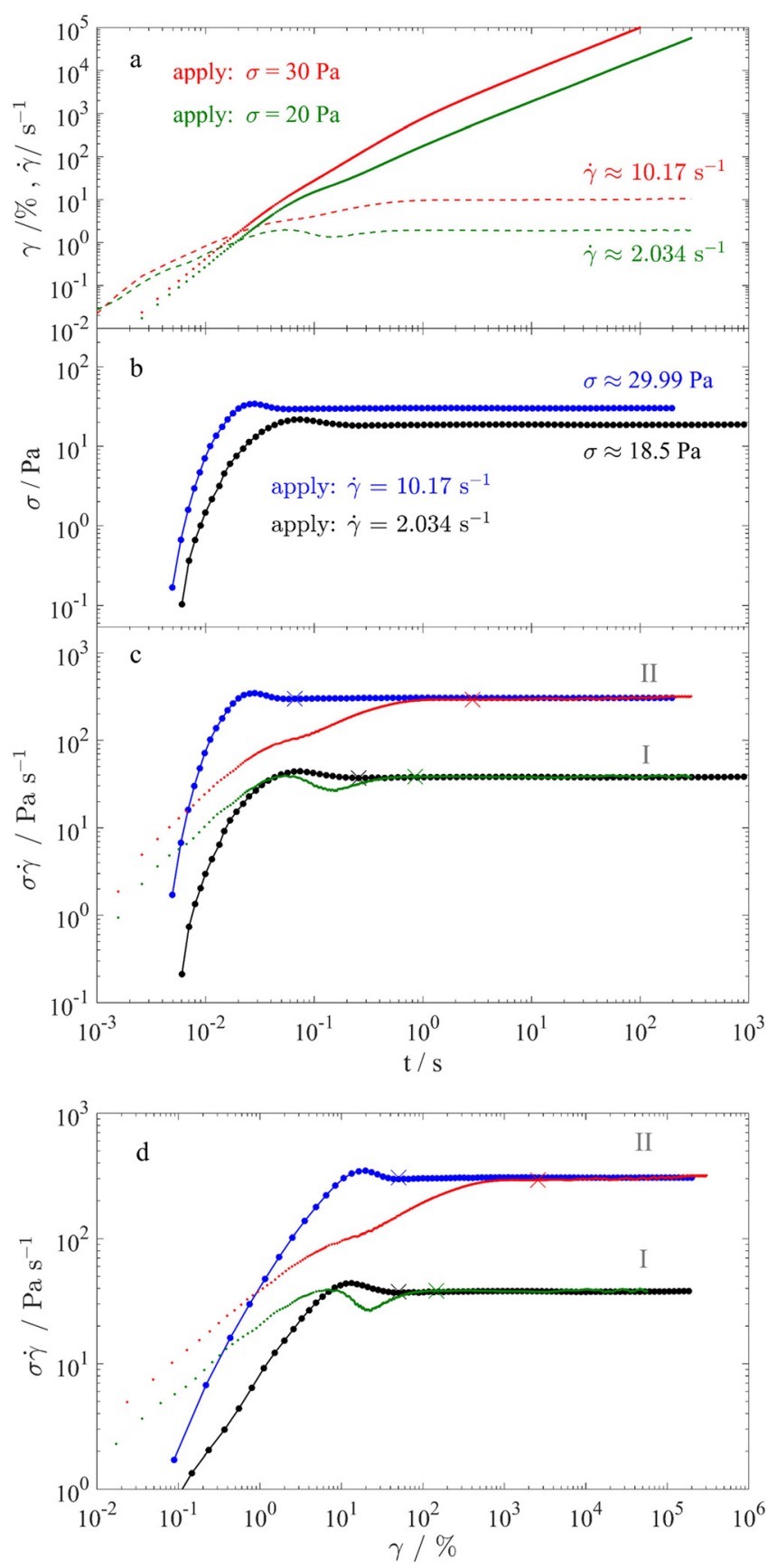

FIG. 8. (a) Strain $\gamma(t)$ (symbols) and corresponding shear rate $\dot{\gamma}(t)$ (a dotted line) as a function of time $t$ measured in creep experiments with stresses $\sigma=20$ and $30 \mathrm{~Pa}$. (b) Stress $\sigma(t)$ as a function of $t$ measured in step-rate experiments with shear rates $\dot{\gamma}=2.0$ and $10.2 \mathrm{~s}^{-1}$. (c) and (d) Data replotted as $\sigma \dot{\gamma}$ as a function of (c) time $t$ and (d) strain $\gamma$. The time $t_{\text {flow }}$ and strain $\gamma_{\text {flow }}$ that are required to reach steady-state flow are indicated by crosses with the corresponding colors. Samples with a size ratio $\delta=0.21$ and composition $x_{\mathrm{s}}=0.7$.

indicating that in this regime the decrease of $t_{\text {flow }}$ is balanced by the increase of the shear rate $\dot{\gamma}$ and hence the decrease of the imposed time scale $1 / \dot{\gamma}$. This suggests that this decrease in $t_{\text {flow }}$ is an effect of the increasing shear rate $\dot{\gamma}$. This shows similarities with observations on yield stress fluids [62]. In contrast, for large $\sigma \dot{\gamma}$ the steady-state flow is reached considerably later by application of a constant stress. This indicates that, in this regime of $\sigma \dot{\gamma}$, application of a constant shear rate leads to a steady state of flow and the fluidization of the

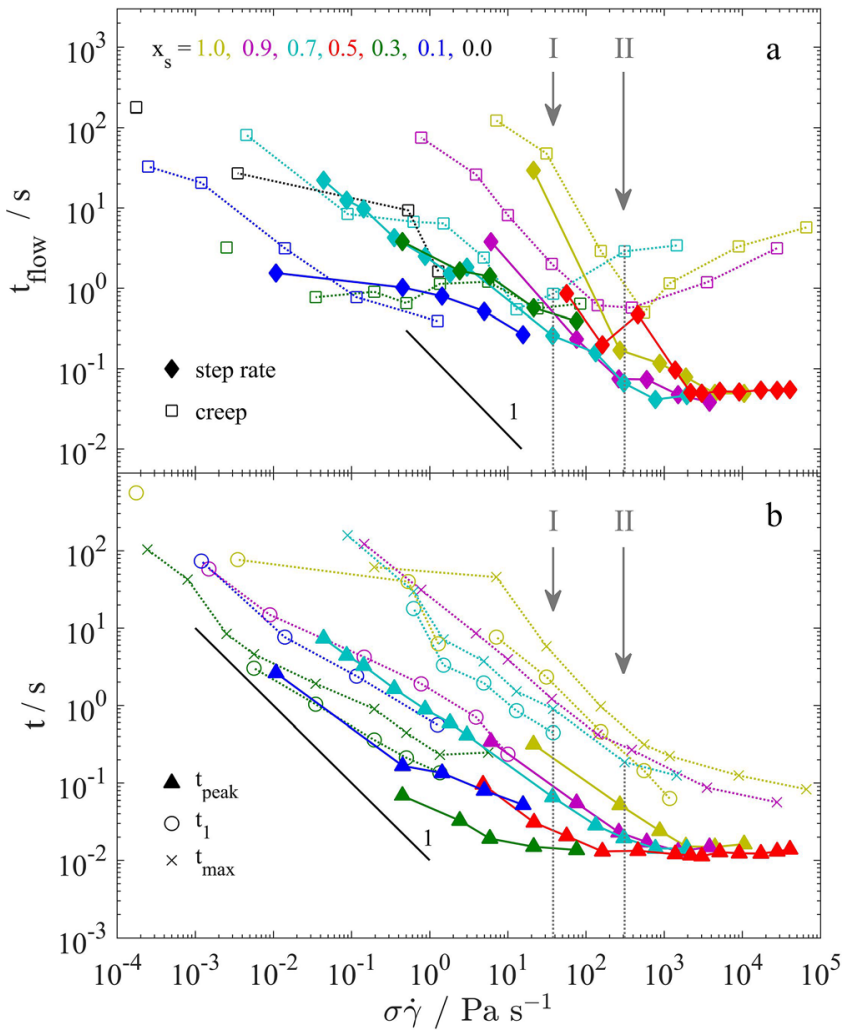

FIG. 9. (a) Time required to reach steady-state flow, $t_{\text {flow }}$, as a function of the product of stress and strain rate, $\sigma \dot{\gamma}$, measured in step-rate $(\diamond)$ and creep $(\square)$ experiments. (b) Time at the stress overshoot, $t_{\text {peak }}(\mathbf{\Lambda})$, measured in step-rate experiments, and time where $\lambda_{\text {creep }}=1, t_{1}(\mathrm{O})$, and the time where $\lambda_{\text {creep }}$ reaches its maximum, $t_{\max }(\times)$, measured in creep experiments as a function of $\sigma \dot{\gamma}$. The conditions shown in Fig. 8 are indicated. Samples with a size ratio $\delta=0.21$ and different compositions $x_{\mathrm{s}}$ (as indicated).

sample in a shorter time. As discussed above (Sec. III B), this might be related to the dominance of shear-induced diffusive and affine motions as well as deformations over Brownian motion under these conditions.

In the transient regime, different characteristic times are relevant; in step-rate experiments the time $t_{\text {peak }}$ when the stress overshoot appears, in creep experiments the time $t_{1}$ when the transition from creep to superlinear behavior occurs (and hence $t_{1}$ is determined by $\lambda_{\text {creep }}=1$ ) and the time $t_{\max }$ when the maximum slope of $\gamma(t)$ is observed (and hence $t_{\max }$ is determined by the maximum of $\lambda_{\text {creep }}$ ) [Fig. 9(b)]. For all compositions $x_{\mathrm{s}}$ and protocols, the characteristic times decrease with increasing $\sigma \dot{\gamma}$ and, at large $\sigma \dot{\gamma}$, become constant. Similar observations have been reported for one-component hard-sphere glasses [14] and colloidal gels [26,52]. Furthermore, for all compositions $x_{\mathrm{s}}$ the characteristic times of the step-rate experiments, $t_{\text {peak }}$, are shorter than those of the creep experiments, $t_{1}$ and $t_{\max }$. This is analogous to the finding that $t_{\text {flow }}$ is slightly shorter in step-rate experiments than in creep experiments. Thus, at comparable values of $\sigma \dot{\gamma}$, in a colloidal glass yielding as well as flow is achieved in a shorter time if a constant shear rate is applied than if a constant stress is applied.

Next we consider the efficiency of the two protocols to achieve yielding or to induce flow. The work per volume, $W$, required to reach yielding or the steady state of flow is given 
by $W=\int_{0}^{t_{\mathrm{y}}} \sigma \dot{\gamma}(t) \mathrm{d} t$ with $t_{\mathrm{y}}=t_{\mathrm{peak}}, t_{1}, t_{\mathrm{max}}$ or $t_{\text {flow }}$ characterizing the time required for yielding or reaching flow. It hence depends on the time-dependent power density, $\sigma \dot{\gamma}(t)$, but also the time required to achieve yielding or flow [Fig. 8(c)]. Since previous work associated the yielding and flow of glasses to the deformation and breakdown of cages [12,19-21] and the loss of long-lived nearest neighbors [63], we interpret the work to reach yielding and flow as the work needed to irreversibly rearrange and break the cage structure in the glass.

To be able to compare creep and step-rate experiments, $W$ to reach flow [Fig. 10(a)] and yielding [Fig. 10(b)], respectively, is shown as a function of $\sigma \dot{\gamma}$. Less work is required to achieve yielding or flow if a step rate is applied. This corresponds to the smaller characteristic times required in steprate experiments. Furthermore, $W$ increases with increasing $\sigma \dot{\gamma}$ indicating that yielding and flow is most efficiently, although slowly, achieved by applying a small stress or strain rate. The increase is very moderate at small values of $\sigma \dot{\gamma}$. This is attributed to the balance between the increase in the applied stress or shear rate and the decrease in the time needed to reach yielding or flow (Fig. 9). In addition, the yield strain or stress typically increase slightly with the applied stress or shear rate, which contributes to the increase

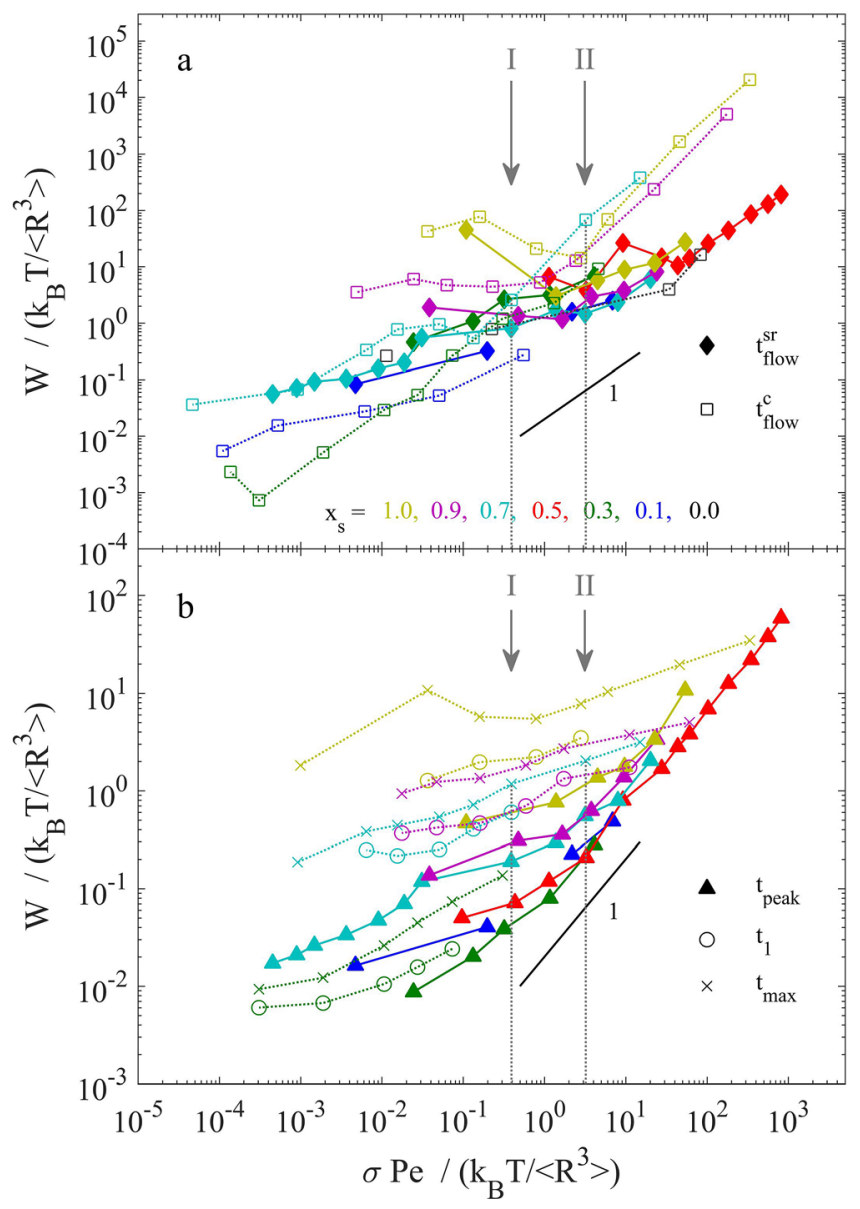

FIG. 10. Work per volume, $W$, required to achieve (a) flow and (b) yielding, respectively, as a function of the product of stress and strain rate, $\sigma \dot{\gamma}$, in the stress- and strain-controlled experiments (as indicated, see text for details). The conditions shown in Fig. 8 are indicated. Samples with a size ratio $\delta=$ 0.21 and different compositions $x_{\mathrm{s}}$ (as indicated). of the required work $W$. At large $\sigma \dot{\gamma}, W$ becomes proportional to $\sigma \dot{\gamma}$ for all compositions $x_{\mathrm{s}}$ with the limiting behavior occurring already at smaller $\sigma \dot{\gamma}$ for weaker glasses. In this regime, fluidization is reached quickly (Fig. 9). This suggests that, beyond a minimum stress or shear rate, flow is induced almost instantaneously and cannot be achieved within a shorter time while the required work still increases, about linearly with $\sigma \dot{\gamma}$ due to the about constant times required to reach yielding and flow. In other words, if the applied stress or shear rate is increased beyond a threshold, the time to yield and reach flow does not significantly decrease any longer and therefore the amount of work increases due to the increased rate of energy input. Hence the process becomes less efficient without any significant gain in time. The absolute scale of $W$, e.g., characterized by the limiting value of $W$ at small $\sigma \dot{\gamma}$, depends on the sample composition $x_{\mathrm{s}}$ and appears to follow the $x_{\mathrm{s}}$ dependence of the shear modulus.

The different characteristic times and work observed in the creep and strain-controlled experiments (Figs. 9 and 10) can be related to the single-particle dynamics [24,31]. In a creep experiment, the MSD of individual particles becomes superdiffusive in the transient regime. At the same time, the strain $\gamma(t)$ increases superlinearly [Fig. 2(a)]. Thus, the MSD is proportional to the strain $\gamma(t)$ [24]. In a step-rate experiment, the MSD also shows transient superdiffusive behavior, about when the stress overshoot occurs [31]. However, due to the constant shear rate, the strain $\gamma(t)$ increases only linearly with time. Hence the dynamics increases faster than the strain $\gamma(t)$ and, therefore, requires less time than in a creep experiment. This is consistent with the yielding and transition to flow which is observed to be reached within a shorter time in step-rate experiments than in creep experiments. Furthermore, it indicates that the enhanced dynamics are responsible for yielding and flow.

\section{CONCLUSIONS}

Using binary mixtures of hard-sphere-like particles, we investigated the rheological response of concentrated fluids and glasses that are characterized by different caging mechanisms. In particular, we compare their responses to the application of a step in stress and a step in shear rate, respectively. While the steady state of flow is identical for both protocols, the transient regimes are different. At comparable values of $\sigma \dot{\gamma}$, yielding and flow is achieved within a shorter time and requires less work if a step rate rather than a step stress is applied. In both cases, larger applied fields, i.e., stresses or strain rates, require more work to achieve yielding and flow but lead to yielding and flow within shorter times, up to a sample-dependent limiting stress or strain rate. Furthermore, the composition, i.e., the glass state and caging mechanism, affects yielding in step-rate experiments [Fig. 7(c)] whereas it seems not in creep experiments (Fig. 4). This might be due to the slower yielding process in creep experiments in which the cage has been found to deform intermittently rather than continuously [15]. This might conceal the effects of the different caging mechanisms. The more efficient yielding and transition to flow as well as the 
shorter times needed to achieve them in step-rate experiments appears to be linked to the dynamics which also is faster in step-rate experiments than under constant stress [24,31]. This is consistent with the expectation that yielding and flow are controlled by the dynamics.

\section{ACKNOWLEDGMENTS}

The authors thank Andrew Schofield (University of Edinburgh) for providing the PMMA particles and Dimitris Vlassopoulos (University of Crete) for very helpful discussions. Furthermore, the authors thank the Deutsche Forschungsgemeinschaft (DFG) for funding through the research unit FOR1394, project P2, and support for T.S. G.P., A.R.J., and J.H. acknowledge support from the EU through the project "ESMI." G.P. and A.R.J. acknowledge funding from the Greek National Funding through Grant THALES “Covisco" and ARISTEIA II "MicroSoft."

\section{References}

[1] Pusey, P. N., "Liquids, freezing and the glass transition," in Les Houches, Session LI, edited by J. P. Hansen, D. Levesque, and J. ZinnJustin (North-Holland, Amsterdam, 1991), pp. 763-942.

[2] Donth, E., The Glass Transition: Relaxation Dynamics in Liquids and Disordered Materials (Springer-Verlag, Berlin, 2001).

[3] Williams, S. R., and W. van Megen, "Motions in binary mixtures of hard colloidal spheres: Melting of the glass," Phys. Rev. E 64, 041502 (2001).

[4] Götze, W., Complex Dynamics of Glass-Forming Liquids: A ModeCoupling Theory (Oxford Science Publications, Oxford, UK, 2012).

[5] Brambilla, G., D. El Masri, M. Pierno, L. Berthier, L. Cipelletti, G. Petekidis, and A. B. Schofield, "Probing the equilibrium dynamics of colloidal hard spheres above the mode-coupling glass transition," Phys. Rev. Lett. 104, 169602 (2010).

[6] Mason, T. G., and D. A. Weitz, "Linear viscoelasticity of colloidal hard sphere suspensions near the glass transition,” Phys. Rev. Lett. 75, 2770-2773 (1995).

[7] Mewis, J., and N. J. Wagner, Colloidal Suspension Rheology (Cambridge University, New York, 2012).

[8] Larson, R. G., The Structure and Rheology of Complex Fluids (Oxford University, New York, 1999).

[9] Pham, K. N., G. Petekidis, D. Vlassopoulos, S. U. Egelhaaf, P. N. Pusey, and W. C. K. Poon, "Yielding of colloidal glasses," Europhys. Lett 75, 624-630 (2006).

[10] Pham, K. N., G. Petekidis, D. Vlassopoulos, S. U. Egelhaaf, W. C. K. Poon, and P. N. Pusey, "Yielding behavior of repulsion- and attraction-dominated colloidal glasses," J. Rheol. 52, 649-676 (2008).

[11] Besseling, R., E. R. Weeks, A. B. Schofield, and W. C. K. Poon, "Three-dimensional imaging of colloidal glasses under steady shear," Phys. Rev. Lett. 99, 028301 (2007).

[12] Koumakis, N., M. Laurati, A. R. Jacob, K. Mutch, A. Abdellali, A. B. Schofield, S. U. Egelhaaf, J. F. Brady, and G. Petekidis, "Start-up shear of concentrated colloidal hard spheres: Stresses, dynamics and structure," J. Rheol. 60, 603-623 (2016).

[13] Petekidis, G., D. Vlassopoulos, and P. N. Pusey, "Yielding and flow of sheared colloidal glasses," J. Phys.: Condens. Matter 16, S3955 (2004).

[14] Siebenbürger, M., M. Ballauff, and Th. Voigtmann, "Creep in colloidal glasses,” Phys. Rev. Lett. 108, 255701 (2012).
[15] Ballesta, P., and G. Petekidis, "Creep and aging of hard-sphere glasses under constant stress," Phys. Rev. E 93, 042613 (2016).

[16] Petekidis, G., A. Moussaid, and P. N. Pusey, "Rearrangements in hardsphere glasses under oscillatory shear strain," Phys. Rev. E 66, 051402 (2002).

[17] Eisenmann, C., C. Kim, J. Mattsson, and D. A. Weitz, "Shear melting of a colloidal glass," Phys. Rev. Lett. 104, 035502 (2010).

[18] Christopoulou, C., G. Petekidis, B. Erwin, M. Cloitre, and D. Vlassopoulos, "Ageing and yield behaviour in model soft colloidal glasses," Phil. Trans. R. Soc. A 367, 5051-5071 (2009).

[19] Koumakis, N., M. Laurati, S. U. Egelhaaf, J. F. Brady, and G. Petekidis, "Yielding of hard-sphere glasses during start-up shear," Phys. Rev. Lett. 108, 098303 (2012).

[20] Mutch, K. J., M. Laurati, C. P. Amann, M. Fuchs, and S. U. Egelhaaf, "Time-dependent flow in arrested states-transient behaviour," Eur. Phys. J. Spec. Top. 222, 2803-2817 (2013).

[21] Zausch, J., J. Horbach, M. Laurati, S. U. Egelhaaf, J. M. Brader, Th. Voigtmann, and M. Fuchs, "From equilibrium to steady state: the transient dynamics of colloidal liquids under shear," J. Phys.: Condens. Matter 20, 404210 (2008).

[22] Laurati, M., K. J. Mutch, N. Koumakis, J. Zausch, C. P. Amann, A. B. Schofield, G. Petekidis, J. F. Brady, J. Horbach, M. Fuchs, and S. U. Egelhaaf, "Transient dynamics in dense colloidal suspensions under shear: shear rate dependence," J. Phys.: Condens. Matter 24, 431207 (2012).

[23] Jacob, A. R., A. S. Poulos, S. Kim, J. Vermant, and G. Petekidis, "Convective cage release in model colloidal glasses," Phys. Rev. Lett. 115, 218301 (2015).

[24] Sentjabrskaja, T., P. Chaudhuri, M. Hermes, W. C. K. Poon, J. Horbach, S. U. Egelhaaf, and M. Laurati, "Creep and flow of glasses: strain response linked to the spatial distribution of dynamical heterogeneities," Sci. Rep. 5, 11884 (2015).

[25] Oswald, P., Rheophysics: The Deformation and Flow of Matter (Cambridge University, Cambridge, 2009).

[26] Divoux, T., C. Barentin, and S. Manneville, "Stress overshoot in a simple yield stress fluid: an extensive study combining rheology and velocimetry," Soft Matter 7, 8409 (2011).

[27] Chaudhuri, P., and J. Horbach, "Onset of flow in a confined colloidal glass under an imposed shear stress," Phys. Rev. E 88, 040301 (2013).

[28] Andrade, E. N., "On the viscous flow in metals, and allied phenomena," Proc. R. Soc. Lond. A 84, 1-12 (1910).

[29] Rosti, J., J. Koivisto, L. Laurson, and M. J. Alava, "Fluctuations and scaling in creep deformation," Phys. Rev. Lett. 105, 100601 (2010).

[30] Koumakis, N., J. F. Brady, and G. Petekidis, "Complex oscillatory yielding of model hard-sphere glasses,” Phys. Rev. Lett. 110, 178301 (2013).

[31] Sentjabrskaja, T., M. Hermes, W. C. K. Poon, C. D. Estrada, R. Castaneda-Priego, S. U. Egelhaaf, and M. Laurati, "Transient dynamics during stress overshoots in binary colloidal glasses," Soft Matter 10, 6546-6555 (2014).

[32] Sentjabrskaja, T., R. Babaliari, J. Hendricks, M. Laurati, G. Petekidis, and S. U. Egelhaaf, "Yielding of binary colloidal glasses," Soft Matter 9, 4524-4533 (2013).

[33] Sentjabrskaja, T., D. Guu, M. P. Lettinga, S. U. Egelhaaf, and M. Laurati, "Glasses of dynamically asymmetric binary colloidal mixtures: Quiescent properties and dynamics under shear," AIP Conf. Proc. 1518, 206 -213 (2013).

[34] Yethiraj, A., and A. van Blaaderen, "A colloidal model system with an interaction tunable from hard sphere to soft and dipolar," Nature $\mathbf{4 2 1}$, 513-517 (2003).

[35] The samples with $\delta=0.21$ were suspended in a mixture of cis-decalin and cycloheptyl-bromide instead of octadecene and bromonaphtalene 
to allow for a comparison with results from confocal microscopy experiments, which require to match the refractive indices of the particles and solvent mixture and which are reported separately [24,31].

[36] Royall, C. P., W. C. K. Poon, and E. R. Weeks, "In search of colloidal hard spheres," Soft Matter 9, 17-27 (2013).

[37] Schaertl, W., and H. Sillescu, "Brownian dynamics of polydisperse colloidal hard spheres: Equilibrium structures and random close packings,” J. Stat. Phys. 77, 1007-1025 (1994).

[38] Koumakis, N., A. Pamvouxoglou, A. S. Poulos, and G. Petekidis, "Direct comparison of the rheology of model hard and soft particle glasses," Soft Matter 8, 4271-4284 (2012).

[39] van Megen, W., T. C. Mortensen, S. R. Williams, and J. Müller, "Measurement of the self-intermediate scattering function of suspensions of hard spherical particles near the glass transition," Phys. Rev. E 58, 6073-6085 (1998).

[40] Wagner, N. J., and J. F. Brady, "Shear thickening in colloidal dispersions," Phys. Today 62(10), 27-32 (2009).

[41] Cheng, X., J. H. McCoy, J. N. Israelachvili, and I. Cohen, "Imaging the microscopic structure of shear thinning and thickening colloidal suspensions," Science 333, 1276-1279 (2011).

[42] van der Vaart, K., Y. Rahmani, R. Zargar, Z. Hu, D. Bonn, and P. Schall, "Rheology of concentrated soft and hard-sphere suspensions," J. Rheol. 57, 1195-1209 (2013).

[43] Le Grand, A., and G. Petekidis, "Effects of particle softness on the rheology and yielding of colloidal glasses," Rheol. Acta 47, 579-590 (2008).

[44] Dinkgreve, M., J. Paredes, M. A. J. Michels, and D. Bonn, "Universal rescaling of flow curves for yield-stress fluids close to jamming," Phys. Rev. E 92, 012305 (2015).

[45] Nordstrom, K. N., E. Verneuil, P. E. Arratia, A. Basu, Z. Zhang, A. G. Yodh, J. P. Gollub, and D. J. Durian, "Microfluidic rheology of soft colloids above and below jamming," Phys. Rev. Lett. 105, 175701 (2010).

[46] Rodriguez, B. E., E. W. Kaler, and M. S. Wolfe, "Binary mixtures of monodisperse latex dispersions. 2. viscosity," Langmuir 8, 2382-2389 (1992).

[47] Lionberger, R. A., "Viscosity of bimodal and polydisperse colloidal suspensions," Phys. Rev. E 65, 061408 (2002).

[48] Farris, R. J., "Prediction of the viscosity of multimodal suspensions from unimodal viscosity data," Trans. Soc. Rheol. 12, 281-301 (1968).

[49] Møller, P. C. F., J. Mewis, and D. Bonn, "Yield stress and thixotropy: On the difficulty of measuring yield stresses in practice," Soft Matter 2, 274-283 (2006).
[50] Coussot, P., H. Tabuteau, X. Chateau, L. Tocquer, and G. Ovarlez, "Aging and solid or liquid behavior in pastes," J. Rheol. 50, 975-994 (2006).

[51] Moller, P., A. Fall, V. Chikkadi, D. Derks, and D. Bonn, "An attempt to categorize yield stress fluid behaviour," Phil. Trans. R. Soc. A 367, 5139-5155 (2009).

[52] Laurati, M., S. U. Egelhaaf, and G. Petekidis, "Nonlinear rheology of colloidal gels with intermediate volume fraction," J. Rheol. 55, 673-706 (2011).

[53] Landrum, B. J., W. B. Russel, and R. N. Zia, "Delayed yield in colloidal gels: Creep, flow, and re-entrant solid regimes," J. Rheol. 60, 783-807 (2016).

[54] Shrivastav, G. P., P. Chaudhuri, and J. Horbach, "Yielding of glass under shear: A directed percolation transition precedes shear-band formation," Phys. Rev. E 94, 042605 (2016).

[55] Moorcroft, R. L., M. E. Cates, and S. M. Fielding, "Age-dependent transient shear banding in soft glasses," Phys. Rev. Lett. 106, 055502 (2011).

[56] Divoux, T., M. A. Fardin, S. Manneville, and S. Lerouge, "Shear banding of complex fluids," Ann. Rev. Fluid Mech. 48, 81-103 (2016).

[57] Ballauff, M., J. M. Brader, S. U. Egelhaaf, M. Fuchs, J. Horbach, N. Koumakis, M. Krüger, M. Laurati, K. J. Mutch, G. Petekidis, M. Siebenbürger, Th. Voigtmann, and J. Zausch, "Residual stresses in glasses,” Phys. Rev. Lett. 110, 215701 (2013).

[58] Amann, C. P., M. Siebenbürger, M. Krüger, F. Weysser, M. Ballauff, and M. Fuchs, "Overshoots in stress-strain curves: Colloid experiments and schematic mode coupling theory," J. Rheol. 57, 149-175 (2013).

[59] Fielding, S. M., "Shear banding in soft glassy materials," Rep. Prog. Phys. 77, 102601 (2014).

[60] Besseling, R., L. Isa, P. Ballesta, G. Petekidis, M. E. Cates, and W. C. K. Poon, "Shear banding and flow-concentration coupling in colloidal glasses," Phys. Rev. Lett. 105, 268301 (2010).

[61] Hendricks, J., R. Capellmann, A. B. Schofield, S. U. Egelhaaf, and M. Laurati, "Different mechanisms for dynamical arrest in largely asymmetric binary mixtures," Phys. Rev. E 91, 032308 (2015).

[62] Divoux, T., D. Tamarii, C. Barentin, and S. Manneville, "Transient shear banding in a simple yield stress fluid," Phys. Rev. Lett. 104, 208301 (2010).

[63] Laurati, M., P. Maßhoff, K. J. Mutch, S. U. Egelhaaf, and A. Zaccone, "Long-lived neighbors determine the rheological response of glasses," Phys. Rev. Lett. 118, 018002 (2017). 